\title{
INVESTIGATION OF THERMOPHYSICAL PROPERTIES OF CARBON TETRACHLORIDE WITH ALKOXYETHANOLS AT 298.15 K-313.15 K.
}

\author{
D. Venkatesan ${ }^{1, *}$ and D. Joshua Amarnath ${ }^{1}$ \\ ${ }^{1}$ Department of Chemical Engineering, \\ Sathyabama Institute of Science and Technology, Chennai-600119, India \\ *E-mail: venkatesaniitkgp@gmail.com
}

\begin{abstract}
In this article, the behavior of carbon tetrachloride +2 -methoxyethanol, carbon tetrachloride +2 -ethoxyethanol and carbon tetrachloride +2 -butoxyethanol are studied. Density and Viscosity of above three binary mixtures have been measured, studied as a function of temperature and composition. Molar volume, Viscosity deviations and Excess Gibb's free energy of activation of flow have been calculated. Excess properties are fit to the Redlich-Kister model to obtain the coefficients and standard error. The results of three binary liquid mixtures signify a strong specific type of interaction between the unlike molecules.
\end{abstract}

Keywords: Density, Viscosity, Excess Properties, Molecular Interactions

(C) RASĀYAN. All rights reserved

\section{INTRODUCTION}

Non-ideal solutions are recognized by determining the strength of the intermolecular forces among the different molecules in particular solution ${ }^{1}$. The data on liquids and liquid mixtures are associated with some of the properties that are finding wide application in solution theory. These findings are useful to study the Thermophysical properties of liquid mixtures and their dependence on temperature and composition. In recent years, the need for the thermophysical properties are raising and it is very significant in different applications, like the design of distillation columns, pipeline systems, and mass transfer operations ${ }^{2}$. Alkoxyethanols, which are oxygenated compounds, are used as additives to gasoline as a result enriching of octane and reducing pollution. The mixture included in this work contains a wide range of applications in industries, as paint thinners, stain removers, solvents and also used in industries as a refrigerant, cleaning fluid, decreasing agent to an extend of insecticides, pesticides, and medicines.

\section{EXPERIMENTAL}

Analytical grade carbon tetrachloride was supplied by S.D. fine chemicals Pvt. Ltd., Mumbai and 2methoxyethanol 2-ethoxyethanol and 2-butoxyethanol were obtained from SRL Pvt. Ltd., Mumbai (India). All the chemicals were degassed prior to use ${ }^{3,4}$. The purities of all the chemicals were compared with literature values and verified. In all cases, the purity was satisfactory to fulfill our requirement and no additional purification was essential. All the binary mixtures, carbon tetrachloride +2 methoxyethanol, carbon tetrachloride +2 -ethoxyethanol and carbon tetrachloride +2 -butoxyethanol were placed in air-tight bottles ${ }^{5}$. The mass measurements were done on a digital electronic balance (Mettler AE 240 , Switzerland) accurate to $0.1 \mathrm{mg}$. The binary mixtures were ready just before use ${ }^{6}$. The pure liquids and liquid mixtures densities were measured with bicapillary pycnometer. The density values are quite accurate and satisfactory. The capillary viscometer was used to measure the viscosity of pure liquids and liquids mixtures with a good accuracy. All the experiments were performed by using a thermostat, which is maintained to $\pm 0.01 \mathrm{~K}^{7}$.

Rasayan J. Chem., 12(2), 589-597(2019)

http://dx.doi.org/10.31788/RJC.2019.1225092

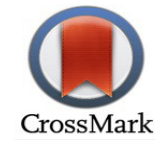




\section{RESULTS AND DISCUSSION}

The pure compounds $\rho$ and $\eta$ values are compared with literature values and presented in Table- 1 . The values observed from Table-1 the experimental values are fairly well with the literature values. The experimentally measured $\rho$ and $\eta$ for studied three binary mixtures at $298.15 \mathrm{~K}-303.15 \mathrm{~K}$ are reported in Table-2.

Table-1: Experimental $\rho$ and $\eta$ Data of Pure Substance, Comparing with Literature Values at 298.15K-313.15 K

\begin{tabular}{|c|c|c|c|c|}
\hline \multirow[t]{2}{*}{$\mathrm{T} / \mathrm{K}$} & \multicolumn{2}{|c|}{ Density $\rho\left(\mathrm{g} . \mathrm{cm}^{-3}\right)$} & \multicolumn{2}{|c|}{ Viscosity $\eta$ (mPa.s) } \\
\hline & Expt. & lit. & Expt. & lit. \\
\hline \multicolumn{5}{|c|}{ Carbon tetrachloride } \\
\hline 298.15 & 1.5837 & $\begin{array}{c}1.5840^{8} \\
1.5839^{14} \\
\end{array}$ & 0.9441 & $0.9443^{14}$ \\
\hline 303.15 & 1.5743 & $\begin{array}{c}1.5748^{8} \\
1.5737^{14} \\
\end{array}$ & 0.8845 & $\begin{array}{l}0.8430^{8} \\
0.8841^{14} \\
\end{array}$ \\
\hline 308.15 & 1.5651 & $\begin{array}{c}1.5650^{8} \\
1.5639^{14}\end{array}$ & 0.8290 & $0.8292^{14}$ \\
\hline 313.15 & 1.5558 & $1.5542^{14}$ & 0.7785 & $0.7788^{14}$ \\
\hline \multicolumn{5}{|c|}{ 2-Methoxyethanol } \\
\hline 298.15 & 0.9605 & $0.9603^{24}$ & 1.5626 & $1.5620^{24}$ \\
\hline 303.15 & 0.9556 & $\begin{array}{l}0.95576^{9} \\
0.9527^{10}\end{array}$ & 1.4716 & $\begin{array}{l}1.4710^{9} \\
1.476^{11}\end{array}$ \\
\hline 308.15 & 0.95117 & $\begin{array}{l}0.95112^{9} \\
0.9503^{10}\end{array}$ & 1.2553 & $\begin{array}{l}1.254^{9} \\
1.257^{12} \\
\end{array}$ \\
\hline 313.15 & 0.9467 & $\begin{array}{l}0.94628^{9} \\
0.9456^{10} \\
\end{array}$ & 1.1626 & $1.162^{9}$ \\
\hline \multicolumn{5}{|c|}{ 2-Ethoxyethanol } \\
\hline 298.15 & 0.9262 & $\begin{array}{l}0.9256^{24} \\
0.9256^{25}\end{array}$ & 1.8545 & $\begin{array}{l}1.850^{24} \\
1.850^{25}\end{array}$ \\
\hline 303.15 & 0.9219 & $\begin{array}{l}0.92117^{9} \\
0.9186^{10}\end{array}$ & 1.6458 & $\begin{array}{l}1.647^{9} \\
1.643^{11}\end{array}$ \\
\hline 308.15 & 0.9167 & $\begin{array}{l}0.91642^{9} \\
0.9160^{10}\end{array}$ & 1.4879 & $\begin{array}{l}1.4870^{9} \\
1.480^{12}\end{array}$ \\
\hline 313.15 & 0.9123 & $\begin{array}{l}0.91225^{9} \\
0.9103^{10}\end{array}$ & 1.2901 & $\begin{array}{l}1.2890^{9} \\
1.293^{11}\end{array}$ \\
\hline \multicolumn{5}{|c|}{ 2-Butoxyethanol } \\
\hline 298.15 & 0.8969 & $\begin{array}{c}0.8966^{24} \\
0.8966^{26} \\
\end{array}$ & 2.7891 & $\begin{array}{l}2.7950^{24} \\
2.7950^{26}\end{array}$ \\
\hline 303.15 & 0.8927 & $\begin{array}{l}0.89213^{9} \\
0.8921^{10}\end{array}$ & 2.4017 & $\begin{array}{r}2.4020^{9} \\
2.4030^{10}\end{array}$ \\
\hline 308.15 & 0.8885 & $\begin{array}{l}0.88705^{9} \\
0.8870^{10}\end{array}$ & 2.2878 & $\begin{array}{r}2.2880^{9} \\
2.2880^{10}\end{array}$ \\
\hline 313.15 & 0.8843 & $\begin{array}{l}0.88224^{9} \\
0.8822^{10}\end{array}$ & 2.1098 & $\begin{array}{l}2.1180^{9} \\
2.1190^{10}\end{array}$ \\
\hline
\end{tabular}

Table-2: The Measured Densities and Viscosities Values at 298.15 K - 313.15 K

\begin{tabular}{c|c|c|c|c|c|c|c|c}
\hline$x_{1}$ & $\begin{array}{c}\rho \\
\left(\mathrm{g} . \mathrm{cm}^{-3}\right)\end{array}$ & $\begin{array}{c}\eta \\
(\mathrm{mPa} . \mathrm{s})\end{array}$ & $\begin{array}{c}\rho \\
\left(\mathrm{g} . \mathrm{cm}^{-3}\right)\end{array}$ & $\begin{array}{c}\eta \\
(\mathrm{mPa} . \mathrm{s})\end{array}$ & $\begin{array}{c}\rho \\
\left(\mathrm{g} . \mathrm{cm}^{-3}\right)\end{array}$ & $\begin{array}{c}\eta \\
(\mathrm{mPa} . \mathrm{s})\end{array}$ & $\begin{array}{c}\rho \\
\left(\mathrm{g} . \mathrm{cm}^{-3}\right)\end{array}$ & $\begin{array}{c}\eta \\
(\mathrm{mPa} . \mathrm{s})\end{array}$ \\
\hline \multicolumn{8}{c}{ Carbon tetrachloride $(1)+2-M e t h o x y e t h a n o l$} \\
\hline \multicolumn{2}{c|}{$(2)$} \\
\hline 0.0000 & 0.9605 & 1.6490 & 0.9561 & 1.4570 & 0.9517 & 1.2920 & \multicolumn{2}{c}{$313.15 \mathrm{~K}$} \\
\hline 0.0831 & 1.0228 & 1.5690 & 1.0181 & 1.3920 & 1.0132 & 1.240 & 1.0084 & 1.1080 \\
\hline 0.1694 & 1.0852 & 1.4900 & 1.0800 & 1.3280 & 1.0747 & 1.1880 & 1.0694 & 1.0670 \\
\hline 0.2591 & 1.1475 & 1.4120 & 1.1418 & 1.2650 & 1.1361 & 1.1370 & 1.1304 & 1.0250 \\
\hline
\end{tabular}


RASĀYAN J. Chem. Vol. 12 | No. 2 |589-597 | April - June | 2019

\begin{tabular}{|c|c|c|c|c|c|c|c|c|}
\hline 0.3523 & 1.2098 & 1.3350 & 1.2037 & 1.2020 & 1.1671 & 1.1110 & 1.1913 & 0.9841 \\
\hline 0.4494 & 1.2721 & 1.2600 & 1.2349 & 1.1710 & 1.2589 & 1.0360 & 1.2522 & 0.9429 \\
\hline 0.5504 & 1.3344 & 1.1860 & 1.3273 & 1.0790 & 1.3202 & 0.9853 & 1.3134 & 0.9015 \\
\hline 0.6557 & 1.3967 & 1.1140 & 1.3891 & 1.0190 & 1.3512 & 0.9601 & 1.3738 & 0.8608 \\
\hline 0.7655 & 1.4589 & 1.0430 & 1.4509 & 0.9601 & 1.4427 & 0.8863 & 1.4345 & 0.8201 \\
\hline 0.8802 & 1.5212 & 0.9736 & 1.5126 & 0.9020 & 1.5039 & 0.8376 & 1.4951 & 0.7796 \\
\hline 1.000 & 1.5837 & 0.9062 & 1.5743 & 0.8450 & 1.5651 & 0.7897 & 1.5558 & 0.7394 \\
\hline \multicolumn{9}{|c|}{ Carbon tetrachloride (1) +2-Ethoxyethanol (2) } \\
\hline & \multicolumn{2}{|c|}{$298.15 \mathrm{~K}$} & \multicolumn{2}{|c|}{$303.15 \mathrm{~K}$} & \multicolumn{2}{|c|}{$308.15 \mathrm{~K}$} & \multicolumn{2}{|c|}{$313.15 \mathrm{~K}$} \\
\hline 0.0000 & 0.9262 & 1.9200 & 0.9219 & 1.6820 & 0.9175 & 1.4790 & 0.9132 & 1.3060 \\
\hline 0.0977 & 0.9903 & 1.7840 & 0.9856 & 1.5720 & 0.9808 & 1.3910 & 0.9760 & 1.2360 \\
\hline 0.1959 & 1.0547 & 1.6570 & 1.0496 & 1.4700 & 1.0444 & 1.3080 & 1.0392 & 1.1680 \\
\hline 0.2946 & 1.1195 & 1.5390 & 1.1140 & 1.3730 & 1.1084 & 1.2300 & 1.1027 & 1.1050 \\
\hline 0.3938 & 1.1847 & 1.4280 & 1.1787 & 1.2830 & 1.1726 & 1.1550 & 1.1665 & 1.0440 \\
\hline 0.4935 & 1.2503 & 1.3250 & 1.2438 & 1.1970 & 1.2372 & 1.0850 & 1.2306 & 0.9864 \\
\hline 0.5937 & 1.3162 & 1.2290 & 1.3092 & 1.1180 & 1.3021 & 1.0190 & 1.2950 & 0.9318 \\
\hline 0.6945 & 1.3824 & 1.1400 & 1.3749 & 1.0430 & 1.3673 & 0.9566 & 1.3597 & 0.8798 \\
\hline 0.7958 & 1.4491 & 1.0560 & 1.4410 & 0.9725 & 1.4329 & 0.8976 & 1.4247 & 0.8306 \\
\hline 0.8976 & 1.5161 & 0.9785 & 1.5075 & 0.9067 & 1.4988 & 0.8421 & 1.4901 & 0.7838 \\
\hline 1.0000 & 1.5837 & 0.9062 & 1.5743 & 0.8450 & 1.5651 & 0.7897 & 1.5558 & 0.7394 \\
\hline \multicolumn{9}{|c|}{ Carbon tetrachloride (1) +2-Butoxyethanol (2) } \\
\hline & \multicolumn{2}{|c|}{$298.15 \mathrm{~K}$} & \multicolumn{2}{|c|}{$303.15 \mathrm{~K}$} & \multicolumn{2}{|c|}{$308.15 \mathrm{~K}$} & \multicolumn{2}{|c|}{$313.15 \mathrm{~K}$} \\
\hline 0.0000 & 0.8969 & 3.5740 & 0.8927 & 3.0990 & 0.8885 & 2.6990 & 0.8843 & 2.3610 \\
\hline 0.1311 & 0.9656 & 2.9860 & 0.9610 & 2.6140 & 0.9564 & 2.2980 & 0.9517 & 2.0280 \\
\hline 0.2534 & 1.0343 & 2.5250 & 1.0293 & 2.2300 & 1.0242 & 1.9770 & 1.0191 & 1.7590 \\
\hline 0.3678 & 1.1030 & 2.1580 & 1.0975 & 1.9220 & 1.0919 & 1.7180 & 1.0863 & 1.5410 \\
\hline 0.4750 & 1.1717 & 1.8620 & 1.1657 & 1.6720 & 1.1596 & 1.5060 & 1.1536 & 1.3600 \\
\hline 0.5758 & 1.2403 & 1.6220 & 1.2339 & 1.4660 & 1.2273 & 1.3300 & 1.2207 & 1.2100 \\
\hline 0.6706 & 1.3090 & 1.4240 & 1.3020 & 1.2960 & 1.2950 & 1.1840 & 1.2879 & 1.0840 \\
\hline 0.7600 & 1.3776 & 1.2600 & 1.3701 & 1.1540 & 1.3626 & 1.0610 & 1.3549 & 0.9770 \\
\hline 0.8445 & 1.4463 & 1.1220 & 1.4382 & 1.0340 & 1.4301 & 0.9560 & 1.4219 & 0.8858 \\
\hline 0.9243 & 1.5149 & 1.0050 & 1.5063 & 0.9323 & 1.4976 & 0.8666 & 1.4889 & 0.8073 \\
\hline 1.0000 & 1.5837 & 0.9062 & 1.5743 & 0.8450 & 1.5651 & 0.7897 & 1.5558 & 0.7394 \\
\hline
\end{tabular}

The properties like molar volume, viscosity deviation and excess Gibbs free energy of activation flow were estimated from the experimental value by using the following equations.

$\mathrm{V}_{\mathrm{m}}=\left(x_{1} \mathrm{M}_{1}+x_{2} \mathrm{M}_{2}\right) / \rho_{\mathrm{m}}$

$\eta^{E}=\eta-\sum_{i=1}^{n} x_{i} \eta_{i}$

$\Delta G^{* E}=R T[\ln (V \eta)]-\sum_{i=1}^{n} x_{i} \ln \left(V_{i} \eta_{i}\right)$

Where $x_{\mathrm{i}}$ represents the mole fraction of the pure component i. $\mathrm{V}, \rho_{\mathrm{m}}$, and $\eta$ are the molar volume, density, and viscosity of liquid mixtures. $V_{i}$, and $\eta_{i}$ the respective properties of the pure substance. The excess properties values are fitted with Redlich-Kister model ${ }^{15}$.

$A^{E}=x_{1}\left(1-x_{1}\right)-\sum_{i=1}^{n} A_{i}\left(2 x_{2}-1\right)^{i}$

The values of $A_{i}$ of Eq. (4) were estimated by using the regression method and the standard deviation values are estimated by the following equation.

$\sigma=\left[\frac{\Sigma\left(x_{\sup }-x_{\text {and }}\right)}{n-p}\right]^{2}$ 
Where $\mathrm{n}$ is the number of measured points, $\mathrm{p}$ is the number of parameters, $x_{\exp }$ and $x_{\mathrm{cal}}$ are the measured and estimated properties.

The viscosity deviation against composition at $298.15 \mathrm{~K}$ to $313.15 \mathrm{~K}$ for binary mixtures of carbon tetrachloride with alkoxyethanols are presented in Fig.-1,2 and 3.The viscosity deviation values all are negative for the system $\mathrm{CCl}_{4}$ with 2- Methoxyethanol , 2- Ethoxyethanol and 2- Butoxyethanol at temperature $298.15 \mathrm{~K}-313.15 \mathrm{~K}$ for the complete range of composition. The obtained values of viscosity deviation differ in the following order 2-Methoxyethanol $<2$-Ethoxyethanol $<2$-Butoxyethanol at all studied temperature. The negative values viscosity deviation indicates that the dispersion forces are dominant. ${ }^{16-18}$ The viscosity deviation $(\Delta \mathrm{y})$ values indicate that there is a survival of dispersion and dipolar forces between dissimilar molecules and associated with the variation in shape and size of the dissimilar molecules. The obtained values suggest that the existence of intermolecular force upon mixing of three binary liquid mixtures.

Table-3: The Calculated Excess Properties Values , $\mathrm{V}_{\mathrm{m}}, \Delta \eta$, and $\Delta \mathrm{G}^{* \mathrm{E}}$ of Binary Mixtures at $298.15 \mathrm{~K}-313.15 \mathrm{~K}$

\begin{tabular}{|c|c|c|c|c|c|c|}
\hline$x_{1}$ & $\begin{array}{c}\Delta \eta \\
(\mathrm{mPa} . \mathrm{s})\end{array}$ & $\begin{array}{c}V_{m} \\
\left(\mathrm{~cm}^{3} / \mathrm{mol}\right) \\
\end{array}$ & $\begin{array}{c}\Delta G^{* E} \\
(\mathrm{~J} / \mathrm{mol})\end{array}$ & $\begin{array}{c}\Delta \eta \\
(\mathrm{mPa} . \mathrm{s})\end{array}$ & $\begin{array}{c}V_{m} \\
\left(\mathrm{~cm}^{3} / \mathrm{mol}\right)\end{array}$ & $\begin{array}{c}\Delta G^{* E} \\
(\mathrm{~J} / \mathrm{mol})\end{array}$ \\
\hline \multicolumn{7}{|c|}{ Carbon tetrachloride (1) +2-Methoxyethanol (2) } \\
\hline & \multicolumn{3}{|c|}{$298.15 \mathrm{~K}$} & \multicolumn{3}{|c|}{$303.15 \mathrm{~K}$} \\
\hline 0.0000 & 0.0000 & 79.2244 & 0.0000 & 0.0000 & 79.5890 & 0.0000 \\
\hline 0.0831 & -0.0182 & 80.7155 & 4.3214 & -0.0141 & 81.0882 & 3.1995 \\
\hline 0.1694 & -0.0331 & 82.2553 & 7.5813 & -0.0253 & 82.6514 & 6.6061 \\
\hline 0.2591 & -0.0445 & 83.8626 & 10.0345 & -0.0334 & 84.2812 & 10.1149 \\
\hline 0.3523 & -0.0523 & 85.5331 & 11.1770 & -0.0394 & 85.9665 & 11.0966 \\
\hline 0.4494 & -0.0552 & 87.2707 & 12.6569 & -0.0410 & 87.7258 & 13.9606 \\
\hline 0.5504 & -0.0542 & 89.0797 & 12.3606 & -0.0412 & 89.5562 & 11.6981 \\
\hline 0.6557 & -0.0480 & 90.9647 & 12.0738 & -0.0367 & 91.4624 & 10.7413 \\
\hline 0.7655 & -0.0374 & 92.9370 & 9.4992 & -0.0284 & 93.4494 & 8.8976 \\
\hline 0.8802 & -0.0216 & 94.9892 & 5.1958 & -0.0163 & 95.5292 & 5.2410 \\
\hline \multirow[t]{2}{*}{1.0000} & 0.0000 & 97.1336 & 0.0000 & 0.0000 & 97.7012 & 0.0000 \\
\hline & \multicolumn{3}{|c|}{$308.15 \mathrm{~K}$} & \multicolumn{3}{|c|}{$313.15 \mathrm{~K}$} \\
\hline 0.0000 & 0.0000 & 79.9569 & 0.0000 & 0.0000 & 80.3283 & 0.0000 \\
\hline 0.0831 & -0.0102 & 81.4803 & 4.0236 & -0.0079 & 81.8682 & 3.2331 \\
\hline 0.1694 & -0.0189 & 83.0590 & 6.6741 & -0.0134 & 83.4706 & 8.1307 \\
\hline 0.2591 & -0.0248 & 84.7041 & 10.2126 & -0.0186 & 85.1312 & 9.5085 \\
\hline 0.3523 & -0.0290 & 86.4116 & 12.0849 & -0.0212 & 86.8613 & 12.6874 \\
\hline 0.4494 & -0.0303 & 88.1857 & 14.4788 & -0.0226 & 88.6576 & 13.7493 \\
\hline 0.5504 & -0.0302 & 90.0378 & 13.2001 & -0.0225 & 90.5040 & 12.0766 \\
\hline 0.6557 & -0.0272 & 91.9655 & 11.7435 & -0.0200 & 92.4810 & 12.2202 \\
\hline 0.7655 & -0.0212 & 93.9806 & 9.3361 & -0.0156 & 94.5178 & 9.7630 \\
\hline 0.8802 & -0.0123 & 96.0819 & 5.2216 & -0.0090 & 96.6474 & 5.7954 \\
\hline \multirow[t]{3}{*}{1.0000} & 0.0000 & 98.2755 & 0.0000 & 0.0000 & 98.8630 & 0.0000 \\
\hline & \multicolumn{6}{|c|}{ Carbon tetrachloride (1) +2-Ethoxyethanol (2) } \\
\hline & \multicolumn{3}{|c|}{$298.15 \mathrm{~K}$} & \multicolumn{3}{|c|}{$303.15 \mathrm{~K}$} \\
\hline 0.0000 & 0.0000 & 97.3030 & 0.0000 & 0.0000 & 97.7568 & 0.0000 \\
\hline 0.0977 & -0.0370 & 97.2868 & 11.2615 & -0.0282 & 97.7508 & 11.2406 \\
\hline 0.1959 & -0.0644 & 97.2755 & 18.2942 & -0.0481 & 97.7481 & 18.4382 \\
\hline 0.2946 & -0.0824 & 97.2596 & 22.5899 & -0.0625 & 97.7398 & 22.8628 \\
\hline 0.3938 & -0.0928 & 97.2402 & 24.7827 & -0.0694 & 97.7352 & 26.7339 \\
\hline 0.4935 & -0.0947 & 97.2181 & 25.9579 & -0.0719 & 97.7262 & 27.9542 \\
\hline 0.5937 & -0.0891 & 97.2012 & 24.8752 & -0.0670 & 97.7209 & 26.7185 \\
\hline 0.6945 & -0.0759 & 97.1890 & 20.0149 & -0.0577 & 97.7192 & 21.3137 \\
\hline 0.7958 & -0.0572 & 97.1677 & 14.2449 & -0.0434 & 97.7139 & 14.4767 \\
\hline 0.8976 & -0.0315 & 97.1514 & 6.2240 & -0.0240 & 97.7056 & 6.7083 \\
\hline 1.0000 & 0.0000 & 97.1336 & 0.0000 & 0.0000 & 97.7012 & 0.0000 \\
\hline
\end{tabular}


RASĀYAN J. Chem.

Vol. 12 | No. 2 |589-597 | April - June | 2019

\begin{tabular}{|c|c|c|c|c|c|c|}
\hline & \multicolumn{3}{|c|}{$308.15 \mathrm{~K}$} & \multicolumn{3}{|c|}{$313.15 \mathrm{~K}$} \\
\hline 0.0000 & 0.0000 & 98.2256 & 0.0000 & 0.0000 & 98.6881 & 0.0000 \\
\hline 0.0977 & -0.0207 & 98.2291 & 11.1405 & -0.0147 & 98.7122 & 11.3971 \\
\hline 0.1959 & -0.0360 & 98.2348 & 19.4130 & -0.0270 & 98.7264 & 19.7414 \\
\hline 0.2946 & -0.0460 & 98.2336 & 23.8462 & -0.0341 & 98.7414 & 25.3566 \\
\hline 0.3938 & -0.0526 & 98.2437 & 27.5049 & -0.0389 & 98.7574 & 28.5630 \\
\hline 0.4935 & $\begin{array}{l}-0.0538 \\
\end{array}$ & 98.2475 & 28.9903 & $\begin{array}{l}-0.0400 \\
\end{array}$ & 98.7744 & 29.7791 \\
\hline 0.5937 & -0.0507 & 98.2538 & 27.1398 & -0.0378 & 98.7925 & 28.7097 \\
\hline 0.6945 & -0.0437 & 98.2624 & 22.3639 & -0.0327 & 98.8116 & 23.8307 \\
\hline 0.7958 & -0.0329 & 98.2662 & 14.4911 & -0.0245 & 98.8318 & 14.9171 \\
\hline 0.8976 & -0.0182 & 98.2728 & 6.7462 & -0.0136 & 98.8465 & 7.0279 \\
\hline \multirow[t]{3}{*}{1.0000} & 0.0000 & 98.2755 & 0.0000 & 0.0000 & 98.8630 & 0.0000 \\
\hline & \multicolumn{6}{|c|}{ Carbon tetrachloride (1) +2-Butoxyethanol (2) } \\
\hline & \multicolumn{3}{|c|}{$298.15 \mathrm{~K}$} & \multicolumn{3}{|c|}{$303.15 \mathrm{~K}$} \\
\hline 0.0000 & 0.0000 & 131.7605 & 0.0000 & 0.0000 & 132.3804 & 0.0000 \\
\hline 0.1311 & -0.2384 & 127.2225 & 12.3656 & -0.1896 & 127.8315 & 12.4735 \\
\hline 0.2534 & -0.3731 & 122.9862 & 21.2074 & -0.2979 & 123.5837 & 21.1001 \\
\hline 0.3678 & -0.4348 & 119.0225 & 26.3283 & -0.3480 & 119.6190 & 26.6375 \\
\hline 0.4750 & -0.4447 & 115.3061 & 27.8935 & -0.3563 & 115.8996 & 29.2431 \\
\hline 0.5758 & -0.4159 & 111.8234 & 28.6787 & -0.3352 & 112.4034 & 27.7993 \\
\hline 0.6706 & -0.3609 & 108.5361 & 26.2087 & -0.2914 & 109.1196 & 25.6004 \\
\hline 0.7600 & -0.2864 & 105.4440 & 22.9349 & -0.2319 & 106.0212 & 21.7862 \\
\hline 0.8445 & -0.1991 & 102.5158 & 16.5972 & -0.1616 & 103.0931 & 15.6545 \\
\hline 0.9243 & -0.1031 & 99.7523 & 7.9103 & -0.0832 & 100.3218 & 8.7654 \\
\hline \multirow[t]{2}{*}{1.0000} & 0.0000 & 97.1336 & 0.0000 & .0000 & 97.7012 & 0.0000 \\
\hline & \multicolumn{3}{|c|}{$308.15 \mathrm{~K}$} & \multicolumn{3}{|c|}{$313.15 \mathrm{~K}$} \\
\hline 0.0000 & 0.0000 & 133.0062 & 0.0000 & 0.0000 & 133.6379 & 0.0000 \\
\hline 0.1311 & -0.1508 & 128.4464 & 12.7988 & -0.1205 & 129.0807 & 12.8131 \\
\hline 0.2534 & -0.2383 & 124.1990 & 21.1208 & -0.1911 & 124.8206 & 20.6327 \\
\hline 0.3678 & $\begin{array}{l}-0.2788 \\
\end{array}$ & 120.2325 & 27.1722 & -0.2236 & 120.8523 & 27.6617 \\
\hline 0.4750 & -0.2860 & 116.5092 & 30.0434 & -0.2307 & 117.1152 & 28.9647 \\
\hline 0.5758 & -0.2696 & 113.0079 & 28.8349 & -0.2173 & 113.6189 & 29.4175 \\
\hline 0.6706 & -0.2346 & 109.7094 & 27.1299 & -0.1895 & 110.3143 & 27.3233 \\
\hline 0.7600 & -0.1869 & 106.6048 & 23.3891 & -0.1515 & 107.2106 & 22.8508 \\
\hline 0.8445 & -0.1307 & 103.6771 & 16.3836 & -0.1058 & 104.2750 & 16.9133 \\
\hline 0.9243 & -0.0676 & 100.9046 & 8.8073 & -0.0548 & 101.4942 & 9.0401 \\
\hline 1.0000 & 0.0000 & 98.2755 & 0.0000 & 0.0000 & 98.8630 & 0.0000 \\
\hline
\end{tabular}

Table-4: Coefficients $\left(A_{k}\right)$ and Standard Deviation $(\sigma)$ of Three Binary Liquid Mixtures at $298.15 \mathrm{~K}-313.15 \mathrm{~K}$

\begin{tabular}{|c|c|c|c|c|c|c|c|}
\hline $\begin{array}{l}\text { Parameter/ } \\
\text { Function }\end{array}$ & $\mathrm{T} / \mathrm{K}$ & $\mathrm{A}_{0}$ & $\mathrm{~A}_{1}$ & $\mathrm{~A}_{2}$ & $\mathrm{~A}_{3}$ & $\mathrm{~A}_{4}$ & $\sigma$ \\
\hline \multicolumn{8}{|c|}{ Carbon tetrachloride (1) +2-Methoxyethanol (2) } \\
\hline \multirow{4}{*}{$\begin{array}{c}\Delta \eta \\
(\mathrm{mPa} . \mathrm{s})\end{array}$} & 298.15 & -0.2212 & 0.0259 & 0.0050 & -0.0077 & $\begin{array}{l}-0.0091 \\
\end{array}$ & 0.0006 \\
\hline & 303.15 & -0.1665 & 0.0136 & -0.0029 & 0.0082 & -0.0016 & 0.0016 \\
\hline & 308.15 & -0.1223 & 0.0113 & -0.0137 & 0.0009 & 0.0146 & 0.0011 \\
\hline & 313.15 & -0.0915 & 0.0070 & 0.0057 & 0.0045 & $\begin{array}{l}-0.0139 \\
\end{array}$ & 0.0016 \\
\hline \multirow{4}{*}{$\begin{array}{c}\Delta G^{* E} \\
(\mathrm{~J} / \mathrm{mol})\end{array}$} & 298.15 & 50.3430 & 5.8339 & 13.0842 & -17.3607 & -16.4821 & 1.1255 \\
\hline & 303.15 & 50.7534 & $\begin{array}{l}-7.6944 \\
\end{array}$ & -4.7442 & 19.4650 & -1.8380 & 3.5453 \\
\hline & 308.15 & 55.9978 & -1.0687 & -29.5076 & 1.4757 & 33.9056 & 2.7043 \\
\hline & 313.15 & 51.8299 & -4.2845 & 22.8112 & 14.3584 & -39.7333 & 4.5207 \\
\hline \multicolumn{8}{|c|}{ Carbon tetrachloride (1) +2-Ethoxyethanol (2) } \\
\hline \multirow{3}{*}{$\begin{array}{c}\Delta \eta \\
(\mathrm{mPa} . \mathrm{s})\end{array}$} & 298.15 & -0.3778 & 0.0479 & -0.0017 & 0.0000 & -0.0053 & 0.0015 \\
\hline & 303.15 & -0.2857 & 0.0313 & 0.0069 & 0.0079 & -0.0225 & 0.0021 \\
\hline & 308.15 & -0.2149 & 0.0201 & 0.0052 & 0.0043 & -0.0112 & 0.0012 \\
\hline
\end{tabular}


RASĀYAN J. Chem.

Vol. 12 | No. 2 |589-597 | April - June | 2019

\begin{tabular}{|c|c|c|c|c|c|c|c|}
\hline & 313.15 & -0.1590 & 0.0172 & -0.0105 & -0.0076 & 0.0205 & 0.0020 \\
\hline \multirow{4}{*}{$\begin{array}{c}\Delta G^{* E} \\
(\mathrm{~J} / \mathrm{mol})\end{array}$} & 298.15 & -2.9334 & 0.0883 & 7.9261 & -0.0788 & -13.7889 & 3.1828 \\
\hline & 303.15 & -0.8303 & -1.2342 & 19.7691 & 10.5082 & -42.7671 & 4.1266 \\
\hline & 308.15 & -1.0693 & -3.8750 & 14.6969 & 8.4331 & -22.1016 & 2.4668 \\
\hline & 313.15 & 2.4638 & 5.1088 & -17.8229 & -18.0825 & 43.4183 & 4.6881 \\
\hline \multicolumn{8}{|c|}{ Carbon tetrachloride (1) +2-Butoxyethanol (2) } \\
\hline \multirow{4}{*}{$\begin{array}{c}\Delta \eta \\
(\mathrm{mPa} . \mathrm{s})\end{array}$} & 298.15 & -1.7627 & 0.3957 & -0.0431 & 0.0086 & -0.0397 & 0.0009 \\
\hline & 303.15 & -1.4148 & 0.2961 & -0.0504 & 0.0131 & 0.0028 & 0.0010 \\
\hline & 308.15 & -1.1364 & 0.2310 & -0.0352 & -0.0026 & 0.0023 & 0.0014 \\
\hline & 313.15 & -0.9152 & 0.1770 & -0.0291 & -0.0024 & 0.0079 & 0.0015 \\
\hline \multirow{4}{*}{$\begin{array}{c}\Delta G^{* E} \\
(\mathrm{~J} / \mathrm{mol})\end{array}$} & 298.15 & 112.725 & 13.8493 & 45.1352 & -7.6513 & -74.0007 & 2.1043 \\
\hline & 303.15 & 115.621 & 2.5890 & -6.1931 & 10.3278 & 10.6045 & 1.8164 \\
\hline & 308.15 & 119.034 & 13.8729 & 1.2788 & -7.7984 & -2.2758 & 2.7206 \\
\hline & 313.15 & 118.734 & 15.5018 & -2.2492 & -8.0542 & 8.5555 & 2.8687 \\
\hline
\end{tabular}
0.0
0.2
$0.4 \quad \mathrm{x} 1 \quad 0.6$
0.8
1.0

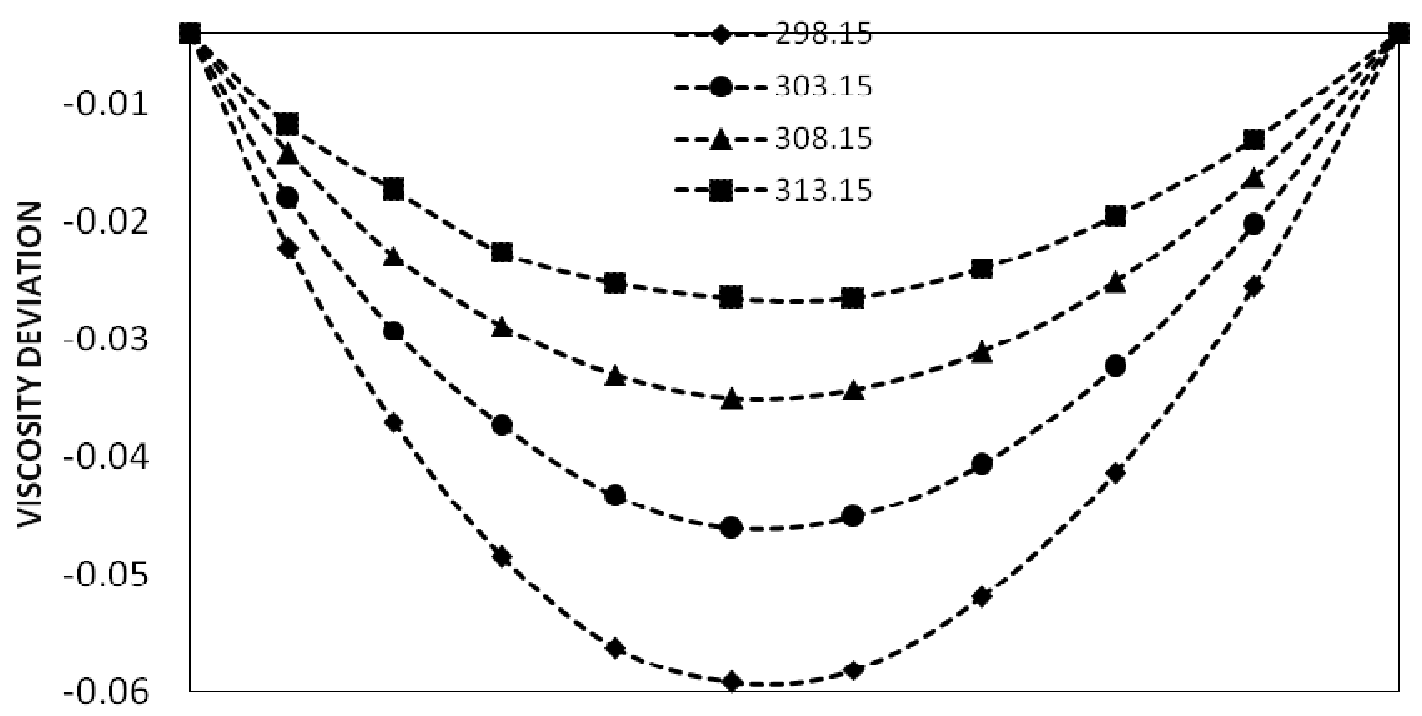

Fig.-1: Viscosity Deviation $(\Delta \eta)$ of $\mathrm{CCL}_{4}(1)+2$ - Methoxy Ethanol (2) vs mole Fraction of $x_{l}$
0.0
0.2
$0.4 \times 1 \quad 0.6$
0.8
1.0

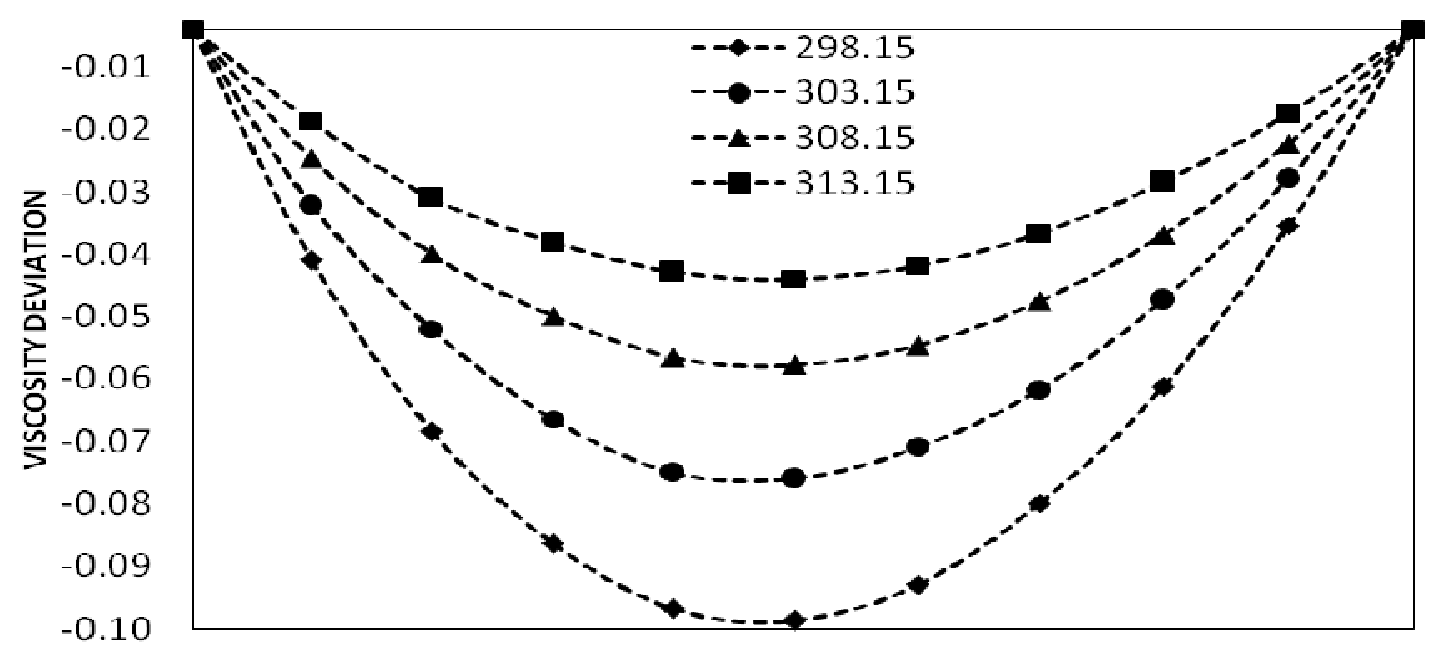

Fig.-2: Viscosity Deviation $(\Delta \eta)$ of $\mathrm{CCL}_{4}(1)+2$-Ethoxy Ethanol (2) vs mole Fraction of $x_{1}$ 
RASĀYAN J. Chem.

Vol. 12 | No. 2 |589 - 597 | April - June | 2019

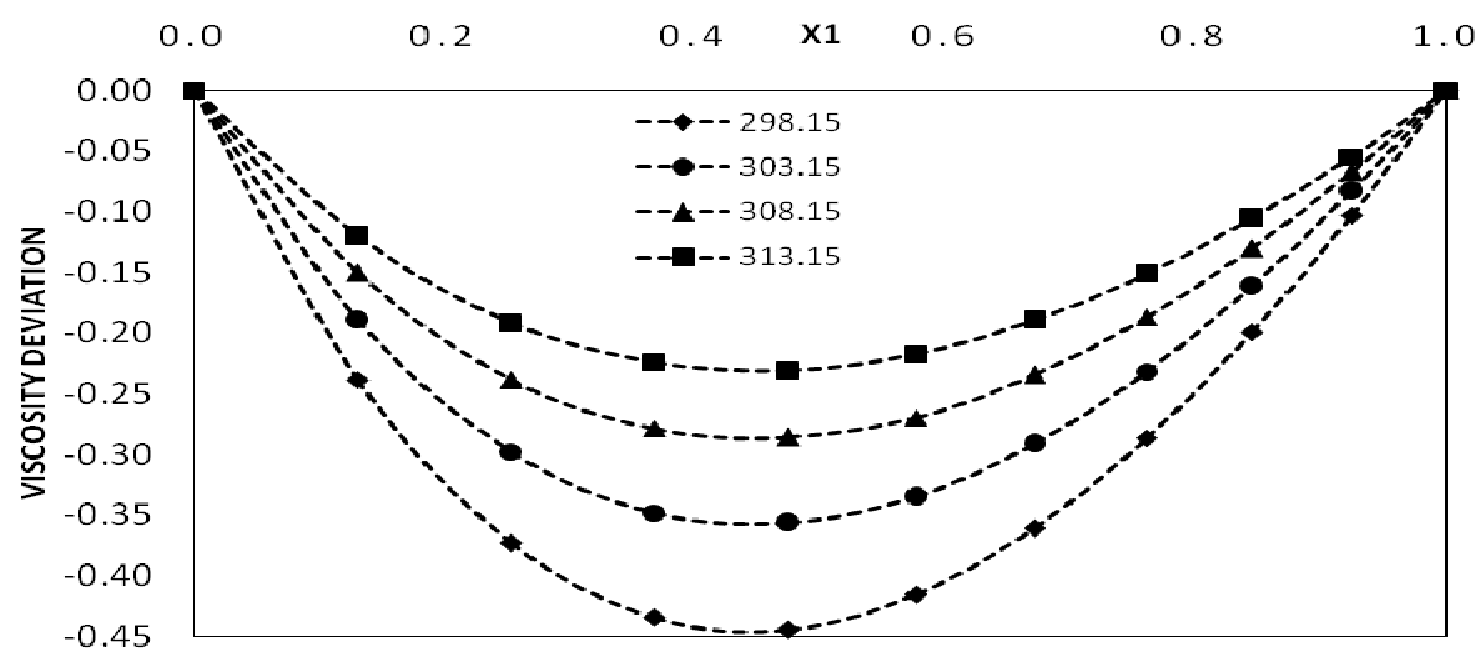

Fig.-3: Viscosity Deviation $(\Delta \eta)$ of $\mathrm{CCL}_{4}(1)+2$-Butoxy Ethanol (2) vs mole Fraction of $x_{1}$

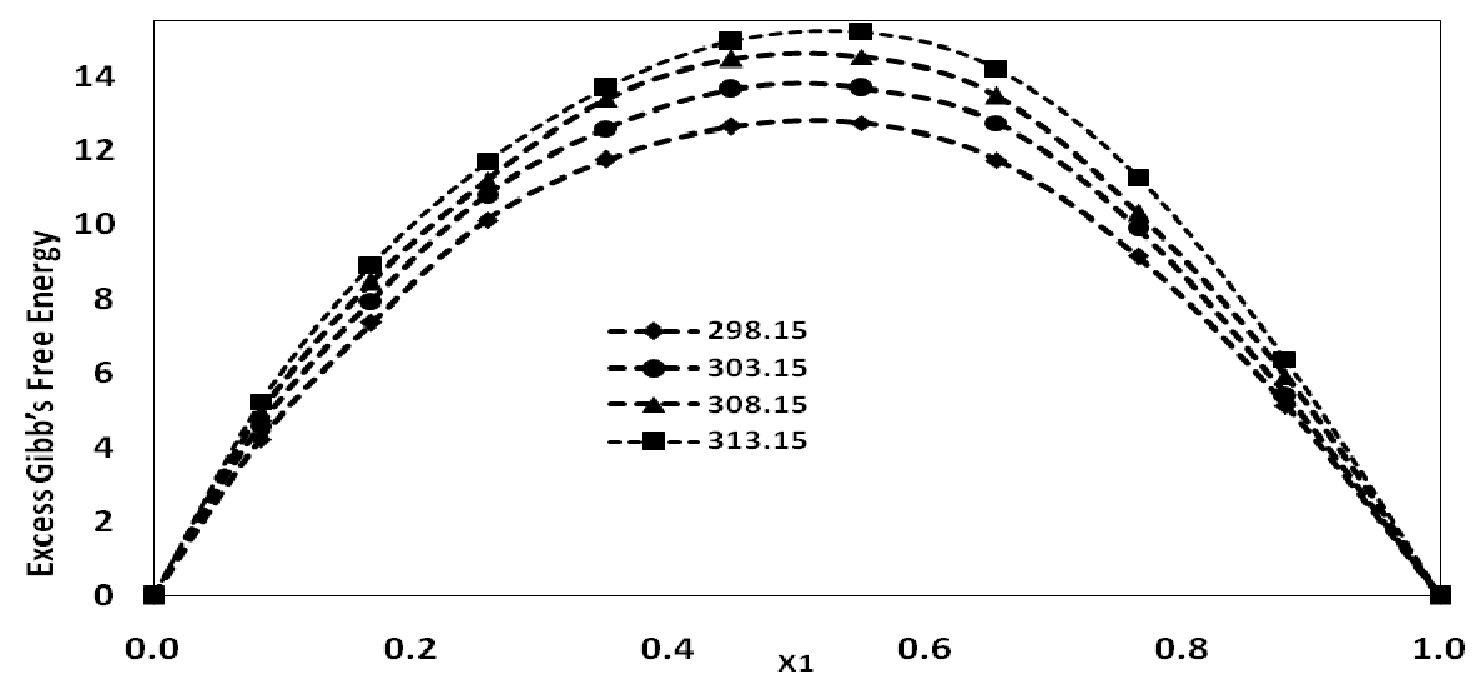

Fig. $-4: \Delta \mathrm{G}^{* \mathrm{E}}$ of CCL4 (1) + 2- Methoxy Ethanol (2) vs mole Fraction of $x_{1}$

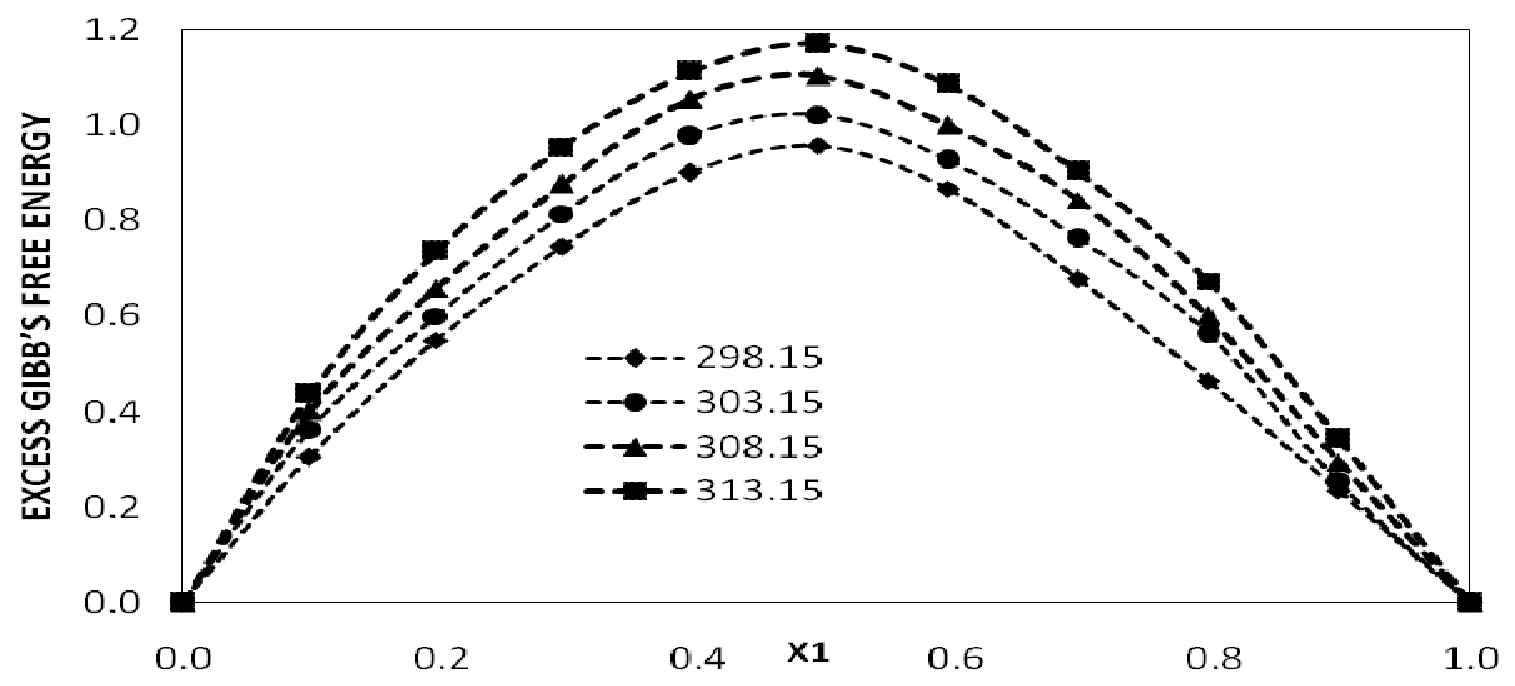

Fig.-5: $\Delta \mathrm{G}^{* \mathrm{E}}$ of $\mathrm{CCL}_{4}(1)+2$ - Ethoxy Ethanol (2) vs mole Fraction of $x_{1}$ 


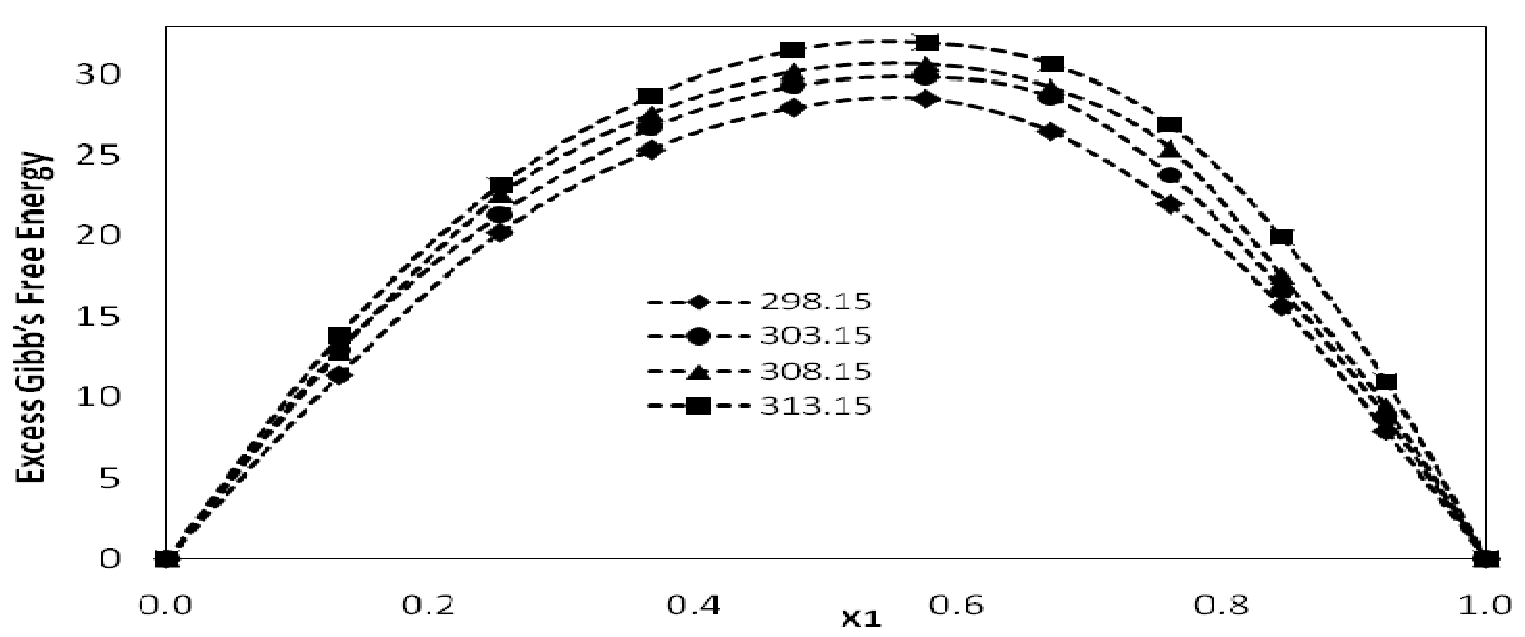

Fig.-6: $\Delta \mathrm{G}^{* \mathrm{E}}$ of $\mathrm{CCL}_{4}(1)+2$-Butoxy Ethanol (2) vs mole Fraction of $x_{1}$

The Excess Gibbs free energy of activation of viscous flow $\left(\Delta \mathrm{G}^{* \mathrm{E}}\right)$ against composition at different temperatures $298.15 \mathrm{~K}$ to $313.15 \mathrm{~K}$ for binary mixtures of $\mathrm{CCl}_{4}$ with alkoxyethanols are presented in Fig.4, 5, and 6. $\Delta \mathrm{G}^{* \mathrm{E}}$ is found to be positive for all mixtures however it initially increases up to 0.6 mole fractions then it gradually decreases. $\Delta \mathrm{G}^{* \mathrm{E}}$ slightly increases by a change in temperature. The values of $\Delta \mathrm{G}^{* \mathrm{E}}$ represents the potency of interaction among dissimilar molecules. ${ }^{19,20}$ The $\Delta \mathrm{G}^{* \mathrm{E}}$ values indicate that there are an exact and strong interaction in the $\mathrm{CCl}_{4}$ with alkoxyethanols. ${ }^{21-23}$ The positive values of $\Delta \mathrm{G}^{*} \mathrm{E}$ for all studied mixtures its show that there is molecular interaction between dissimilar molecules.

\section{CONCLUSION}

Thermophysical properties like Densities and viscosities of three binary liquid mixtures are reported. The values of the pure substance mostly concur with the existing literature. Molar volumes, viscosity deviations and excess Gibb's free energy of activation of a flow of carbon tetrachloride + 2methoxyethanol, carbon tetrachloride +2 -ethoxyethanol and carbon tetrachloride +2 -butoxyethanol were obtained at $T=(298.15,303.15,308.15$ and 313.15) $\mathrm{K}$ from the experimental results and it has reported. The excess properties are fitted with Redlich-Kister equations. The results are analyzed in terms of molecular interactions between the components. The investigation studies of three binary liquid mixtures show that there is an intermolecular interaction between the components.

$\rho$ - Density $\left(\mathrm{g} / \mathrm{cm}^{3}\right)$

\section{NOMENCLATURE}

$\eta$ - Dynamic Viscosity (mPa.s)

$\mathrm{V}_{\mathrm{m}}$ - molar volume $\left(\mathrm{cm}^{3} / \mathrm{mol}\right)$

$\Delta \eta$ - Viscosity Deviation (mPa.s)

$\Delta \mathrm{G}^{* \mathrm{E}}$ - Excess gibbs free energy of activation flow $(\mathrm{J} / \mathrm{mol})$

\section{REFERENCES}

1. A. Mchaweh, A. Alsaygh, Kh. Nasrifar and M. Mosh Feghian, Fluid Phase Equilib., 224,157(2004), DOI: $10.1016 /$ j.fluid.2004.06.054.

2. D.Venkatesan, D. Joshua Amarnath, J. Aravind Kumar, Kiran Baru Chandra and S. Balasubramanian, International Journal of Ambient Energy,1(2018), DOI:10.1080/01430750.2018.1557545.

3. S. R. Patil, U.G. Deshpande and A. R. Hiray, Rasayan J. Chem., 3, 66(2010).

4. K. Saravanakumar, D. Venkatesan and M. Gokulakrishnan, Research Journal of Pharmaceutical, Biological and Chemical Sciences, 5,1392 (2014)

5. D. Venkatesan, D. Joshua Amarnath, and K. Saravanakumar, Research Journal of Pharmaceutical, Biological and Chemical Sciences, 9,143 (2018) 
6. K. Saravanakumar, T. G. Lavanya, R. Baskaran and T. R. Kubendran , J. Iran Chem. Soc., 277,290 (2012)

7. D. Venkatesan and D. Joshua Amarnath, Rasayan J. Chem., 11, 834(2018), DOI: $10.31788 /$ RJC.2018.1122094.

8. Tejraj M. Aminabhavi and Kamalika Banerjee, J. Chem. Eng. Data, 43,1096(1998), DOI: $10.1021 /$ je980145+

9. R. Balaji, M. Gowri Sankar and M. Chandra Shekar, Journal of Molecular Liquids, 216, 330 (2016), DOI: $10.1016 /$ j.molliq.2015.12.051.

10. P. Jeevanandham S. Kumar, and P. Periyasamy, Journal of Molecular Liquids, 188,203(2013), DOI: 10.1016/j.molliq.2013.09.035.

11. S. Kumar and P. Jeevanandham, Journal of Molecular Liquids, 174, 34(2012), DOI: 10.1016/j.molliq.2012.07.025

12. Mahendra Nath Roy, Bipul Kumar Sarkar, and Riju Chanda, J. Chem. Eng. Data, 52 ,1630(2007), DOI: $10.1021 /$ je $700026 \mathrm{~d}$.

13. R. R. Zoting, K. V. Pawar and U. E. Choudhari, Rasayan J. Chem., 7,410(2014).

14. Vidya A. Aminabhavi, Tejraj M. Aminabhavi, and Ramachandra H. Balundgi, Ind. Eng. Chem, 29, 2016 (1990), DOI: 10.1021/ie00106a020.

15. O. Redlich and A. T. Kister, Ind. Eng. Chem.,40, 345 (1948), DOI:10.1021/ie50458a036.

16. M. Aminabhavi Tejraj and Kamalika Banerjee, J. Chem. Eng. Data, 43,1096(1998), DOI:10.1021/je980145+

17. M. V. Rathnam and Sudhir Mohite, J. Chem. Eng. Data, 50,325 (2005), DOI:10.1021/je0400052

18. Surabhi Varshney and Mukhtar Singh, J. Chem. Eng. Data, 51, 1136(2006), DOI: 10.1021/je0600303.

19. Ezekiel Dixon Dikio, Orient. J. Chem., 30,953(2014), DOI:10.13005/ojc/300306.

20. Bal Raj Deshwal, Anu Sharma and Krishan Chander Singh, Chin. J. Chem. Eng., 16, 599(2008), DOI:10.1016/S1004-9541(08)60127-5.

21. John George and Nandhibatla V. Sastry, J. Chem. Eng. Data, 48, 1529(2003), DOI: $10.1021 / \mathrm{je} 0340755$.

22. Ch. Praveen Babu, G. Pavan Kumar and K. Samatha, Int. J. Adv. Sci. Tech., 76,27(2015), DOI: 10.14257/ijast.2015.76.04.

23. Arvind R. Mahajan and Sunil R. Mirgane, Journal of Thermodynamics, 25,75(2013), DOI: $10.1155 / 2013 / 571918$

24. Lovely Sarkar and Mahendra Nath Roy, J. Chem. Eng. Data, 54, 3307(2009), DOI: $10.1021 / \mathrm{je} 900240 \mathrm{~s}$.

25. I. Jhonson, M. Kalidoss, and R. Srinivasamoorthy, J. Chem. Eng. Data, 47, 1388(2002), DOI: $10.1021 / \mathrm{je} 900240 \mathrm{~s}$

26. Anjali AwasthI and Aashees Awasthi, Physics and Chemistry of Liquids, 51, 112 (2013), DOI: 10.1080/00319104.2012.690569

[RJC-5092/2018] 